\title{
Sensor inalámbrico con WiFi energizado por gradientes térmicos para la monitorización de radiadores en ambientes urbanos
}

\author{
Carlos Andrés Trasviña Moreno, Roberto Casas Nebra, Rubén Blasco Marín \\ Human Openware Research Lab (HOWLab) \\ Instituto de Investigación en Ingeniería de Aragón (I3A) \\ Universidad de Zaragoza, Mariano Esquillor s/n, 50018, Zaragoza, Spain. \\ Tel.+34-976762707, e-mail: ctrasvina@humanopenware.com
}

\begin{abstract}
En el presente documento se propone la realización de un sensor WiFi para monitorización de radiadores, con el afán de controlar el consumo energético en el hogar. Dicho dispositivo contará con autonomía energética a través de un elemento de Peltier.
\end{abstract}

\section{Introducción}

Hoy en día existe una gran necesidad por tener un mayor control sobre el consumo energético en el hogar. Uno de los elementos de mayor aporte y menor control en esta área son los radiadores [1], los cuales requieren ser regulados según las últimas normativas europeas [5].

Para esto se busca desarrollar un dispositivo con el cual el usuario pueda monitorizar el consumo individual de sus radiadores, de manera intuitiva y en tiempo real. Para esto se ha pensando en la realización de un sensor que pueda transmitir datos de temperatura de manera inalámbrica a una base datos de fácil acceso. Siendo que en el hogar es común encontrar una infraestructura de comunicaciones WiFi, se decidió emplear este protocolo para la transmisión de datos. Como característica adicional, se busca dejar atras las fuentes de alimentación convensionales, como baterías alcalinas y/o recargables, por energías ambientales renovables.

Para esto se realizaron distintos análisis en las áreas de electrónica de bajo consumo y energy harvesting, las cuales se describen en la siguiente sección.

\section{Metodología}

\section{Electrónica de bajo consumo}

Para poder lograr una verdadera autonomía energética, es necesario que los componentes electrónicos a seleccionar se encuentren optimizados para bajo consumo energético. Esto significa que tendrán, como mínimo, modalidades de dormir y activo, donde se podrá reducir el consumo hasta los nA.

Adicionalmente, se requiere implementar estrategias de bajo consumo en el firmware del dispositivo [4]. Estas estrategias dependerán fuertemente del tipo de protocolo de comunicaciones, así como el módulo de radio seleccionados, ya que no todos se encuentran optimizados para el uso de una u otra estrategia.

Para el actual diseño se propone la utilización de un PIC24FJ256GA106 y un módulo de WiFi-b MRF24W0BMA. Estos dispositivos, de acuerdo a sus hojas de especificaciones, presentan modalidades de consumo de tipo dormir, donde su consumo puede llegar a reducirse haste el orden de los nano amperios.

Posteriormente se decidió diseñar el firmware en base a una estrategia de medición y transmisión bajo demanda, es decir que se realizarían estas actividades bajo ciertos intervalos previamente definidos (Tabla 1).

Habiendo previamente medido los tiempos de sensado y transmisión que le toma al dispositivo sensor, es posible calcular la carga que será requerida para un intervalo de 24 horas de operación continua por parte del bloque de energy harvesting (Tabla 1).

\section{Energy Harvesting}

Dentro del área de energy harvesting se encuentran diversas fuentes ambientales de las cuales es posible extraer energía, sin embargo no todas resultan favorables para uso en interiores. Las que presentan mayores densidades energéticas en este tipo de ambientes son la energía lumínica y térmica [3]. Tomando en cuenta que el objeto a analizar es una fuente de radiación térmica, y considerando las densidades energéticas previamente mensionadas, se 
decidió utilizar este mismo para la generación de energía del dispositivo sensor.

Debido a que la energía emitida por el radiador variará en magnitud a lo largo del tiempo, la cantidad de energía a aprovechar será muy intermitente. Es por esto que se deberá diseñar una etapa de acondicionamiento, almacenamiento y gestión energética, con la cual poder obtener el máximo potencial de nuestra fuente ambiental [2]. Con las estimaciones de consumo del dispositivo sensor, así como las expectativas energéticas por parte del bloque de energy harvesting, se deberá adecuar el tamaño del reservorio energético para poder proveer alimentación de manera continua, aún cuando se este generando poca energía

Para esta implementación se seleccionó un elemento Peltier para su uso como un generador termoeléctrico, al cual se le adhirió un disipador de calor para maximizar su eficiencia. Posteriormente la salida del Peltier se conectaría a un integrado con un convertidor step-up y gestionador de energía con salidas configurables a distintos voltajes. Como reservorio se seleccionó un supercondensador, el cual es capaz de soportar el consumo, del dispositivo sensor, de tipo impulso [2]. Esta etapa se implemento y probó en un ambiente controlado de laboratorio para presentar los resultados expuestos en la Figura 1.

\section{Conclusiones}

En el presente abstract se propone el diseño de un dispositivo sensor WiFi con energy harvesting térmico, con la finalidad de auditar radiadores de hogar en tiempo real. Con las comprobaciones teóricas del consumo del sensor inalámbrico, así como los resultados de la experimentación en laboratorio del módulo de harvesting, es posible comprobar que una implementación de este tipo resulta viable bajo distintos escenarios con un esquema de sensado y tranmisión bajo demanda.

\section{REFERENCIAS}

[1]. GUERRA SANTIN, O. Behavioural Patterns and User Profiles Related to Energy Consumption for Heating. Energy and Buildings. 2011, 43(10). 26622672.

[2]. PENElla, M.T., AlBeSA, J. and GASUlla, M. Powering Wireless Sensor Nodes: Primary Batteries Versus Energy Harvesting. In: IEEE Instrumentation and Measurement Technology Conference, 2009. I2MTC '09. Piscataway: IEEE, 2009, pp. 1625-1630.

[3]. TAN, Y.K. and PANDA, S.K. Energy Harvesting from Hybrid Indoor Ambient Light and Thermal Energy Sources for Enhanced Performance of Wireless Sensor Nodes. IEEE Transactions on Industrial Electronics. 2011, 58(9), 4424-4435.

[4]. TRASVINA-MORENO, C.A., et al. WiFi Sensor Networks: A Study of Energy Consumption. In: 2014 11th International Multi-Conference on Systems, Signals \& Devices (SSD). Piscataway: IEEE, 2014, pp. 1-6.

[5]. UNION EUROPEA. Directiva 2012/27/UE del Parlamento Europeo y del Consejo, de 25 de octubre de 2012, relativa a la eficiencia energética. Diario Oficial de la Unión Europea L 315, 14 de Noviembre de 2012, pp. 1-56.

\section{Tabla 1 - Escenarios de Prueba}

\begin{tabular}{|c|c|c|c|}
\hline $\begin{array}{c}\text { Escenarios } \\
\#\end{array}$ & $\begin{array}{c}\text { Sensar } \\
\text { cada }\end{array}$ & $\begin{array}{c}\text { Transmitir } \\
\text { cada }\end{array}$ & Carga \\
\hline 5 & $600 \mathrm{~s}$ & $600 \mathrm{~s}$ & $20.2 \mathrm{C}$ \\
\hline 6 & $10 \mathrm{~s}$ & $600 \mathrm{~s}$ & $22.2 \mathrm{C}$ \\
\hline 7 & $600 \mathrm{~s}$ & $24 \mathrm{~h}$ & $6.96 \mathrm{C}$ \\
\hline 8 & $60 \mathrm{~s}$ & $4 \mathrm{~h}$ & $7.74 \mathrm{C}$ \\
\hline 9 & $600 \mathrm{~s}$ & $3 \mathrm{~h}$ & $7.61 \mathrm{C}$ \\
\hline
\end{tabular}

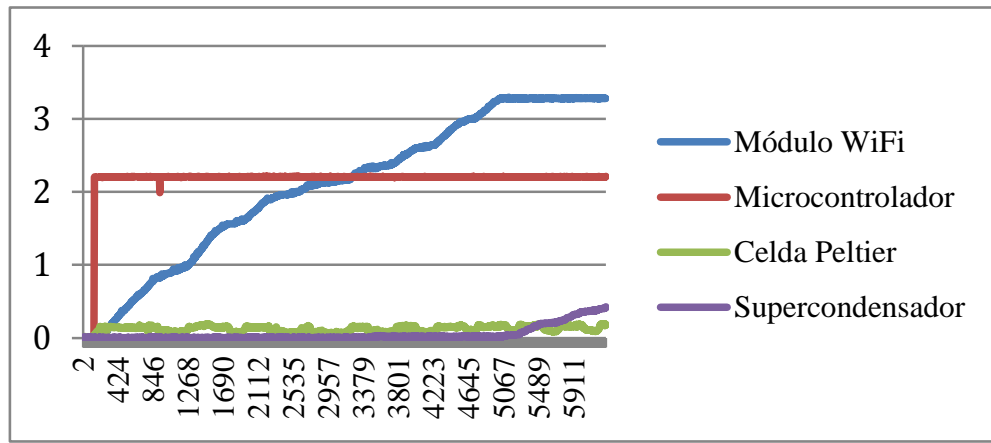

Figura 1 - Gráfico de Voltaje (v) vs Tiempo (s) 